\title{
Developing an Advanced Life Support System for the Flexible Path into Deep Space
}

\author{
Harry W. Jones ${ }^{*}$ and Mark H. Kliss ${ }^{\dagger}$ \\ NASA Ames Research Center, Moffett Field, CA, 94035-0001
}

Long duration human missions beyond low Earth orbit, such as a permanent lunar base, an asteroid rendezvous, or exploring Mars, will use recycling life support systems to preclude supplying large amounts of metabolic consumables. The International Space Station (ISS) life support design provides a historic guiding basis for future systems, but both its system architecture and the subsystem technologies should be reconsidered. Different technologies for the functional subsystems have been investigated and some past alternates appear better for flexible path destinations beyond low Earth orbit. There is a need to develop more capable technologies that provide lower mass, increased closure, and higher reliability. A major objective of redesigning the life support system for the flexible path is achieving the maintainability and ultra-reliability necessary for deep space operations.

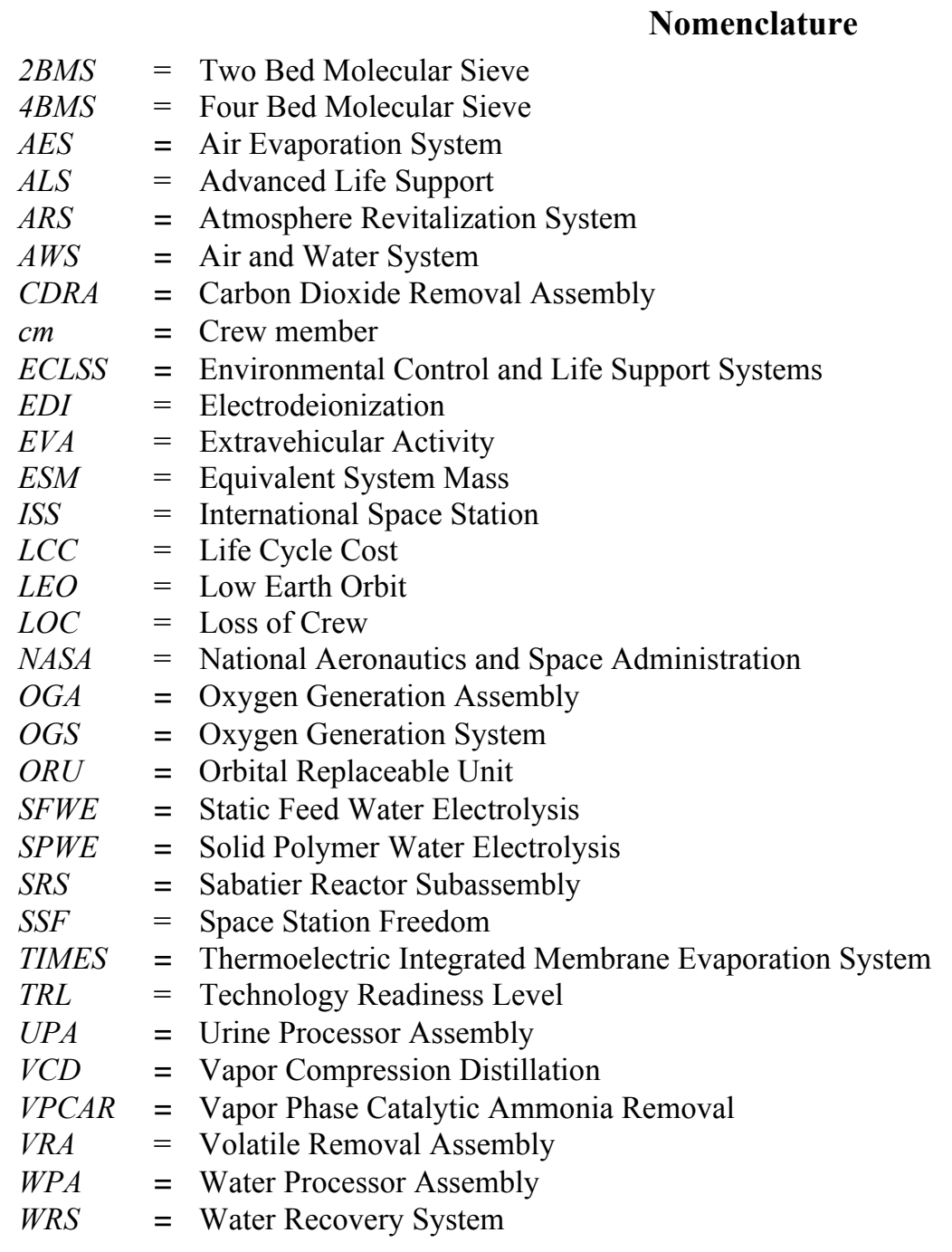

\footnotetext{
* Systems Engineer, Bioengineering Branch, Mail Stop N239-8, AIAA Senior Member.

${ }^{\dagger}$ Chief, Bioengineering Branch, Mail Stop N239-15, AIAA Member.
} 


\section{Introduction}

$\mathrm{C}$ andidate destinations being proposed for flexible path human exploration include the Moon, Lagrange points, asteroids and comets, and Mars. Several possible scenarios require a life support system capable of operating in deep space. With the suggestion that Mars is the ultimate destination, life support technologies being developed for flexible path missions may well be expected to address many of the requirements of the most challenging destinations. Directly supplying all the consumables needed for crew consumption would be extremely expensive and potentially mission limiting for future human missions on the flexible path into deep space. Accordingly, regenerative life support systems are indispensable for human exploration of deep space. Providing additional supplies or spare parts, or returning the crew quickly in the event of a failure, are not possible during many of the potential missions beyond low Earth orbit (LEO). Therefore, flexible path life support systems must be ultrareliable.

This paper proposes the development and long term test of a highly maintainable and reliable recycling life support system for flexible path missions. This next generation life support system would build on the knowledge gained from the current International Space Station (ISS) system but go far beyond it in operational capability by providing a more closed, robust, reliable, maintainable, and adaptable system needed to enable a flexible path of human exploration into deep space. This suggested approach discusses the design, development, test, and demonstration of ultra-reliable life support for deep space. The life support requirements, systems architecture, ISS implementation, and possible candidate technology alternates are described, as is the need for long duration testing to assess the reliability of components and prove out the integrated system.

\section{Why not use the ISS life support system as it is?}

The ISS life support system development and deployment has been a significant achievement. It was designed appropriately to support crew in LEO and it performs most of the life support functions that will be required in the flexible path scenarios. The ISS life support system, however, was designed for an environment where regular resupply from Earth is a key aspect of planned operations, and where in the event of a significant failure, emergency return can be performed rapidly. Because this is not possible on deep space transits or planetary explorations, the life support system must be much more maintainable, repairable, and reliable.

Although human metabolic requirements change little, the driving requirements for life support systems for missions on the flexible path differ significantly from those for ISS in LEO. Flexible path systems have a much stronger design driver to reduce mass, because moving mass beyond LEO significantly increases its cost per kilogram. The amount of water provided to the crew for hygiene will be minimized, and may be much lower than the amount originally planned for the space station. Providing dehydrated food to save up-mass may remove a large current source of water and require greater efficiency in recycling water. Wastes then must be considered as water sources. Reducing the mass of the life support system itself will save much more cost beyond LEO. The high cost of mass beyond LEO drives the life support system to have lower mass and higher recycling efficiencies than the ISS system.

Other driving requirements changes are forced by the greater distance and possible planetary location of the flexible path life support systems. The deep space life support system must operate in several gravity conditions, support Extravehicular Activity (EVA), and tolerate dust contamination. It also should be able to take advantage of in situ resources and accommodate planetary protection measures.

\section{Life support requirements}

Historically, key life support functions have included air revitalization (carbon dioxide removal, oxygen generation), and water reclamation (potable and hygiene water processing, urine reclamation). A summary of the atmosphere and water processing rate requirements for a crewmember is given in Table 1 . 
Table 1. Life support system outputs and inputs, $\mathrm{kg}$ per crewmember per day $(\mathrm{kg} / \mathrm{cm}-\mathrm{d})$.

\begin{tabular}{|l|r|l|r|}
\hline \multicolumn{1}{|c|}{ Output products } & $\mathrm{kg} / \mathrm{cm}-\mathrm{d}$ & \multicolumn{1}{c|}{ Input waste streams } & $\mathrm{kg} / \mathrm{cm}-\mathrm{d}$ \\
\hline Crew oxygen & 0.84 & Carbon dioxide & 1.00 \\
\hline Drinking and food preparation water & 2.37 & Respiration and perspiration condensate & 2.28 \\
\hline Urine flush water & 0.50 & Urine and flush water & 2.00 \\
\hline Wash water & 1.29 & Used wash water & 1.29 \\
\hline Total air and water system products & 5.00 & Total air and water system inputs & 6.57 \\
\hline
\end{tabular}

These are minimal atmosphere and water rate requirements. They are based on space station analysis, except that showers, dish washing, and most hygiene water have been eliminated. (Jones and Kliss, 2005) (Reed and Coulter) (Wieland) The waste streams include carbon dioxide and water produced by the crew from food.

\section{A. Life support mass flows and balances}

A regenerative flexible path life support system will generate most of the needed output products of Table 1 by recycling exhaled carbon dioxide, respiration and perspiration condensate, urine and urine flush water, and used wash water, the waste streams of Table 1 . To some extent the output and input streams can be matched. Oxygen can be derived from water generated as a byproduct of the reduction of carbon dioxide. Drinking and food preparation water can be produced from humidity condensate. Urine flush water can be derived from urine and urine flush water. Wash water can be recycled separately. A summary of the mass flows and system balance in terms of water is shown in Table 2. (Jones and Kliss, 2005)

Table 2. Life support system mass flows and system mass balance, $\mathrm{kg} / \mathrm{cm}-\mathrm{d}$.

\begin{tabular}{|l|r|r|r|}
\hline & $\begin{array}{c}\text { Air and water } \\
\text { system output, } \\
\mathrm{H}_{2} \mathrm{O}, \mathrm{kg} / \mathrm{cm}-\mathrm{d}\end{array}$ & $\begin{array}{c}\text { Air and water } \\
\text { system input, } \\
\mathrm{H}_{2} \mathrm{O}, \mathrm{kg} / \mathrm{cm}-\mathrm{d}\end{array}$ & $\begin{array}{c}\text { Air and water } \\
\text { system input - } \\
\text { output, } \mathrm{H}_{2} \mathrm{O}, \\
\mathrm{kg} / \mathrm{cm}-\mathrm{d}\end{array}$ \\
\hline $\begin{array}{l}\text { Crew and leakage } \\
\text { oxygen }\end{array}$ & 0.95 & & \\
\hline $\begin{array}{l}\text { Crew carbon } \\
\text { dioxide }\end{array}$ & & 0.82 & -0.13 \\
\hline $\begin{array}{l}\text { Drinking and food } \\
\text { preparation water }\end{array}$ & 2.37 & & \\
\hline $\begin{array}{l}\text { Respiration and } \\
\text { perspiration water }\end{array}$ & & & \\
\hline Urine flush water & 0.50 & & -0.09 \\
\hline $\begin{array}{l}\text { Urine and flush } \\
\text { water }\end{array}$ & & & \\
\hline Wash water & 1.29 & 2.00 & \\
\hline Used wash water & & & 0.00 \\
\hline $\begin{array}{l}\text { Overall input - } \\
\text { output }\end{array}$ & 5.11 & 6.39 & 1.29 \\
\hline
\end{tabular}

Table 1 shows that there is a requirement for $0.84 \mathrm{~kg}$ oxygen per crewmember per day and that the crew produces $1.00 \mathrm{~kg}$ of carbon dioxide. Table 2 shows the amount of water corresponding to these amounts of oxygen and carbon dioxide, so water is used as a common accounting basis. The net loss is due to an assumed crew respiratory quotient of less than one. Even if all the oxygen in the carbon dioxide is recovered, additional oxygen must be provided by the electrolysis of $0.13 \mathrm{~kg}$ of water per crewmember per day. Assuming the crew receives 2.37 $\mathrm{kg}$ of drinking and food preparation water per crewmember per day, the condensate of respiration and perspiration amounts to $2.28 \mathrm{~kg}$ of water per crewmember per day, a further loss of $0.09 \mathrm{~kg}$ of water per crewmember per day.

The air and water system supplies $0.50 \mathrm{~kg}$ of urine flush water per crewmember per day, and receives $1.50 \mathrm{~kg}$ of urine and $0.50 \mathrm{~kg}$ of used urine flush water, for a total of $2.00 \mathrm{~kg}$ per crewmember per day. The $1.50 \mathrm{~kg}$ gain is due to $1.15 \mathrm{~kg}$ water in the fully hydrated crew food and $0.35 \mathrm{~kg}$ of water produced by metabolism of the food, both per crewmember per day. $0.22 \mathrm{~kg}$ of the urine and flush water must be purified to make up for the loss of crew oxygen and water. $1.28 \mathrm{~kg}$ of unneeded urine and flush water per crewmember per day must be stored or disposed of. Significantly, the excess water slightly exceeds the water in the fully hydrated food. If dehydrated foods are used to reduce launch mass and mission cost, the water balance will be additionally impacted. 
Although it is possible to treat the waste streams separately, the ISS life support systems architecture combines treated urine and flush water with other wastewater for final processing. Separately treating urine and flush water would probably not significantly increase system costs.

\section{B. Other requirements and design drivers}

The deep space life support system design must consider many requirements and design drivers, including cost, performance, technology readiness, and safety. Life support design has distinct and often conflicting goals. Some design drivers are shown in Table 3.

Table 3. Life support design drivers.

\begin{tabular}{|l|l|}
\hline \multicolumn{1}{|c|}{ Factor } & \multicolumn{1}{c|}{ Metrics or factors } \\
\hline Safety & \# of high criticality failures, Loss of Crew (LOC) \\
\hline Readiness & Technology Readiness Level (TRL) \\
\hline Performance & Product quantity and quality, recycling efficiency \\
\hline 'ilities & Maintainability, reliability, complexity, flexibility \\
\hline Other & Microgravity sensitivity, contamination potential, noise, commonality \\
\hline Cost & Equivalent System Mass (ESM), Life Cycle Cost (LCC) \\
\hline
\end{tabular}

Safety can be measured by the number of high criticality failures, or other metrics such as the probability of Loss of Crew (LOC). Readiness is usually measured by the Technology Readiness Level (TRL). The system performance can be measured by the quantity and quality of the output product and the recycling efficiency. The 'ilities include maintainability, reliability, complexity, and flexibility. Maintainability and reliability are especially important for deep space systems. Other system performance factors include microgravity sensitivity, contamination potential, noise, and commonality. (Jones, 1999-01-2079)

Cost can be measured either by the Equivalent System Mass (ESM) or the Life Cycle Cost (LCC). The Equivalent System Mass (ESM) includes the mass of the flight hardware, the equivalent mass of the spacecraft pressurized volume the hardware uses, and the mass of the power and cooling capacity required to operate the hardware. The pressurized volume is converted to mass using a mass equivalence factor, so many kilograms per cubic meter, based on the launch mass and volume of the structure. The power and cooling requirements are similarly converted to mass at so many kilograms per watt, based on the mass and capacity of the power and cooling systems. The mass of resupplied materials and spare parts is added if they are significant. The LCC estimates all the costs incurred for a system during the development, launch and emplacement, and operations phases. (Jones, 200301-2635)

\section{Reliability}

Deep space life support must have much higher reliability than the ISS life support in LEO, because a deep space mission cannot be resupplied or aborted quickly.

Suppose we achieve an ECLSS reliability of 0.99 for a ten-day shuttle mission to ISS. The corresponding reliability for one day is 0.999 , indicating an expected failure rate of one in a thousand per day. The reliability over a 1,000 day mission would be $0.99^{\wedge} 1,000 / 10=0.37$, so that there would be a 63 percent chance of one or more failures. This is not satisfactory life support reliability for flexible path scenarios.

To achieve a life support reliability of 0.99 for a 1,000-day deep space mission, the corresponding reliability for one day is 0.99999 , indicating an expected failure rate of one in one hundred thousand per day. The reliability over a brief 10-day mission is 0.9999 , a failure rate of one in ten thousand.

Designing a new system that will be reliable for a thousand days will be extremely challenging. Demonstrating such reliability requires long term testing, including in the space environment. Several cycles of design, long duration test, and redesign will be almost certainly needed to achieve sufficiently high reliability for flexible path missions.

\section{ESM}

For deep space missions, we must launch to LEO the deep space vehicle and the propulsion system needed to get the deep space vehicle to its destination and back to LEO. The calculation of the total payload mass ratio in LEO considers the final payload returned to LEO, the second stage return rocket and propellant, and the first stage LEO to deep space rocket and propellant. For a Moon orbit return to LEO, the initial/payload mass ratio is roughly 20. For Mars orbit and return to LEO, the total to payload mass ratio is roughly 50. (Jones, 2003-01-2635) These mass ratios are representative of the flexible path. 
The Space Shuttle cost to launch to LEO is typically quoted as about $\$ 25 \mathrm{k} / \mathrm{kg}$. (Wertz and Larson) The mass increase ratios indicate that we need to launch to LEO propellant that is 20 to 50 times as massive as the deep space vehicle. Based on Space Shuttle launch costs to LEO of $\$ 25 \mathrm{k} / \mathrm{kg}$, the deep space and LEO return costs are $\$ 500$ to $\$ 1,250 \mathrm{k} / \mathrm{kg}$.

Accordingly, the design of the life support system for deep space should place a much higher premium on developing and integrating a low mass, low ESM system. This much higher mass cost does not change the mission duration for recycling to breakeven, since resupply and recycling mass both have the same cost, but it greatly increases the cost saved by using the lower mass recycling approach.

\section{E. Closure}

Although the ISS life support system was initially designed to recycle urine and waste water and to recover oxygen from carbon dioxide, these capabilities have become available only recently with the launch of the water processor and oxygen generation systems, and require resupplied consumables such as filters and contaminant absorbing beds. (Carasquillo, Bagdigian, et al.). Carbon dioxide reduction will likely be demonstrated on ISS for the first time during 2010. Because the ISS has usually been supplied with water from the shuttle fuel cells, closure was not operationally required. So although regenerative life support systems are being demonstrated on ISS, they do not operate with high efficiency and minimal resupply. Systems operating beyond LEO will not have the shuttle or a similar water source, and all supplies will have a high mass launch cost, so a higher recycling efficiency and a higher degree of closure are needed for the flexible path than for ISS.

\section{ISS Life Support System}

In this section the systems architecture of the ISS life support system is described. The selected subsystem technologies and their original ISS alternates are listed. The ISS system represents the current state-of-the-art for the design of recycling life support systems for the flexible path.

\section{A. ISS ECLSS architecture}

Figure 1 shows the ISS life support system, which is the major part of the Environmental Control and Life Support System (ECLSS). (Diamant and Humphries) (Carasquillo and Bertotto) (Bagdigian and Ogle)

Figure 1. The ISS life support system. 


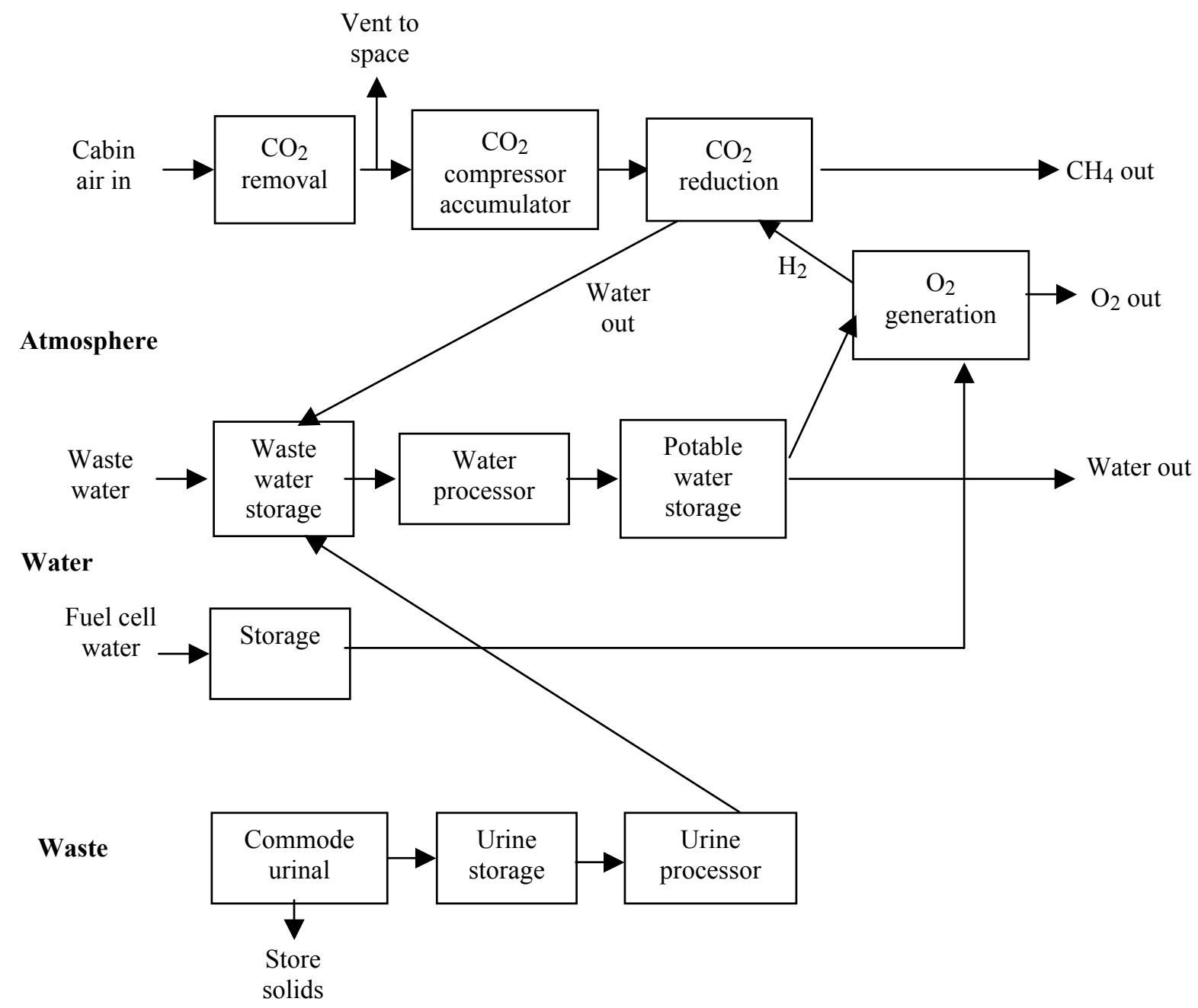

As shown in Figure 1, the ISS life support system contains atmosphere, water, and waste processors. The four bed molecular sieve (4BMS) carbon dioxide removal system is designed to allow the carbon dioxide to be vented to space or to be delivered to the Sabatier carbon dioxide reduction system. The electrolysis oxygen generator provides oxygen directly to the cabin atmosphere. The hydrogen can be vented overboard or used for carbon dioxide reduction.

Waste hygiene water and cabin condensate is stored and routed through the potable water processor to a potable storage tank. Fuel cell water from the Shuttle is stored elsewhere and can be used as needed. Urine is pumped from the urinal to the urine processor and the distillate is combined with other wastewater. The commode bags and compacts feces.

\section{B. ISS life support technologies}

The ISS ECLSS is the only complete recycling life support system design to be flown. The ISS ECLSS systems, assemblies, and technologies are listed in Table 4. 
Table 4. ISS ECLSS technologies.

\begin{tabular}{|c|c|c|}
\hline ISS system & ISS assembly & ISS technology \\
\hline \multirow{2}{*}{$\begin{array}{c}\text { Atmosphere Revitalization } \\
\text { System (ARS) }\end{array}$} & $\begin{array}{c}\text { Carbon Dioxide Removal } \\
\text { Assembly (CDRA) }\end{array}$ & $\begin{array}{c}\text { Four Bed Molecular } \\
\text { Sieve (4BMS) }\end{array}$ \\
\cline { 2 - 3 } & $\begin{array}{c}\text { Sabatier Reactor } \\
\text { Subassembly (SRS) }\end{array}$ & Sabatier \\
\hline $\begin{array}{c}\text { Oxygen Generation System } \\
\text { (OGS) }\end{array}$ & $\begin{array}{c}\text { Oxygen Generation } \\
\text { Assembly (OGA) }\end{array}$ & $\begin{array}{c}\text { Solid Polymer Water } \\
\text { Electrolysis (SPWE) }\end{array}$ \\
\hline \multirow{2}{*}{$\begin{array}{c}\text { Water Recovery System } \\
\text { (WRS) }\end{array}$} & $\begin{array}{c}\text { Water Processor } \\
\text { Assembly (WPA) }\end{array}$ & Multifiltration \\
\cline { 2 - 3 } & Urine Processor & $\begin{array}{c}\text { Vapor Compression } \\
\text { Assembly (UPA) }\end{array}$ \\
\hline
\end{tabular}

The drinking and hygiene water is produced by a multifiltration processor that receives the condensate, hygiene wastewater, and urine distillate. Multifiltration is followed by a Volatile Removal Assembly (VRA) to remove organics. Make-up water has been provided from the space shuttle fuel cell product water and by Progress module resupply. (Carrasquillo, Bagdigian et al.) (Bagdigian and Ogle) (Reuter and Reysa)

In summary, the ISS ECLSS provides most of the functions required for flexible path life support. The ISS ECLSS, however, was not designed to address the additional challenges of operating self-sufficiently for long periods of time far from Earth. It was designed to utilize the earlier selected Space Station Freedom (SSF) technology to the maximum extent possible. (Carrasquillo, Bagdigian et al.) All the ISS technologies, except for SPWE, are the same as those chosen for SSF, which had SFWE (Static Feed Water Electrolysis). Accordingly, alternate technologies and integrated system designs selected to specifically address the new deep space drivers would likely be more effective for flexible path purposes and destinations.

\section{Alternate ISS and SSF technologies}

Since the selection of the baseline and alternate technologies for SSF, further studies have investigated alternate technologies for space station evolution, for the Space Exploration Initiative, and for a new approach to ISS. (Jones and Kliss, 2005) Table 5 shows the ISS and SSF technologies, the SSF alternates, the suggested non-SSF alternates, and the minimum ESM technologies.

Table 5. ISS, SSF, non-SSF alternate, and the minimum ESM technologies.

\begin{tabular}{|c|c|c|c|c|}
\hline & ISS/SSF & SSF alternate & Non-SSF alternates & Minimum ESM \\
\hline Carbon dioxide removal & 4BMS & & 2BMS & 2BMS \\
\hline Carbon dioxide reduction & Sabatier & Bosch & CO2 electrolysis & Sabatier \\
\hline Oxygen generation & SPWE/SFWE & SPWE & WVE, CO2 electrolysis & SFWE \\
\hline Water processing & Multifiltration & RO & EDI & Multifiltration \\
\hline Urine processing & VCD & TIMES & VPCAR, AES & VPCAR \\
\hline
\end{tabular}

Six different non-SSF alternate technologies were recommended by the different studies, as shown in the fourth column of Table 5. Five of the ISS and SSF technologies or alternates (Sabatier, Bosch, SPWE, Multifiltration, and RO) were also recommended by the subsequent studies that considered space station evolution and alternates.

The non-SSF technologies generally are very familiar. They typically have a TRL of 4 or 5 , which is significantly lower than that of the ISS and SSF technologies, but they have demonstrated "development potential." The ESM for the non-SSF alternate selections is not better than for the ISS and SSF selections, except for 2BMS. Carbon dioxide electrolysis provides oxygen directly from carbon dioxide, in a departure from the standard systems architecture. Unfortunately the mass balance and flow still require the capability to generate oxygen from water. The alternate non-SFF technologies are plausible but not clearly superior to the SSF candidates.

Because the mass emplacement cost beyond LEO is much higher than at LEO, technology that minimizes ESM should be used. The ISS technologies' ESM may be reduced up to one half by substituting 2BMS for 4BMS, SFWE for SPWE, and VPCAR for VCD. The Sabatier and multifiltration planned or used in ISS have lower ESM than their technology alternates.

\section{The ISS ECLSS design process}

The ISS ECLSS design accomplished challenging goals under very difficult circumstances, but no design process is guaranteed to produce a perfect product. The ISS ECLSS used SSF technology to the maximum extent 
possible. The technology selection process and results for SSF are reported in a 1992 NASA Technical Memorandum.

"The selection process began during the Space Station Freedom (S.S. Freedom) phase B program with early identification of candidate technologies for regenerative air revitalization (carbon dioxide removal, carbon dioxide reduction, and oxygen generation) and water reclamation (potable and hygiene water processing and urine reclamation) functions. Trade studies conducted by MSFC and the phase B prime contractors narrowed the field of candidate technologies by first identifying those technologies which were not sufficiently mature to meet S.S. Freedom program schedules; such technologies were dropped from further consideration. Trade studies compared the remaining technologies with regard to factors such as safety, resource requirements, performance, reliability, maintenance, and complexity. The basis for these studies were the limited data available from government- and contractor-sponsored development testing which existed at that time. The studies formed the basis for the selections of baseline systems at the end of phase B. To minimize technical risk to the program, parallel development of baseline and alternate subsystems continued to be pursued." (Carrasquillo, Carter, et al.)

The ISS and SSF ECLSS design process can be summarized as follows.

1) Candidate SSF technologies were identified.

2) Technologies of low technical readiness were eliminated.

3) Trade studies using safety, resource requirements, performance, reliability, maintenance, and complexity were used to select baseline and alternate candidates for each SSF technology.

4) The baseline and alternate SSF candidate technologies had prototypes developed and were compared using quantitative and qualitative criteria.

5) The ISS technology selection was the same as the SSF selection, except the chosen SSF baseline oxygen generation technology was later replaced by its SSF alternate technology. (Jones and Kliss, 2005)

This ISS ECLSS design process raises several issues. The first technology screening eliminated low TRL technologies. Although schedule pressure seems an unrealistic justification now, given the many years that elapsed before flight, selecting for high technical maturity did reduce development cost and risk. But technologies that at the time had lower TRL may be capable of improving system performance, as may new technologies currently being researched and developed.

The ISS ECLSS was not designed using alternate system architectures that were traded-off at the system level. The component technologies were traded head-to-head within a predefined system architecture. An alternate ECLSS architecture, for example using carbon dioxide electrolysis or separate water loops, may be competitive.

Not all the ISS technology selections are strongly determined. The candidate technology pairs were compared qualitatively and in detail based on prototype testing, but no overall single figure of merit was developed. 4BMS was selected for carbon dioxide removal without considering any alternate. Sabatier was selected for carbon dioxide reduction because it has much lower mass than the Bosch alternate. The other three technologies chosen for ISS (oxygen generation, water purification, urine purification) were selected based more on qualitative than quantitative justifications. All three selections changed between SSF baseline identification and ISS selection. The three SSF baseline technologies not selected by ISS appear highly competitive with the three alternates that were selected. (Jones and Kliss, 2005) When considering the increased need for system closure and self-sufficiency, a deep space life support design may well select different technologies than those used on ISS.

\section{E. Proposed ISS design upgrades}

Significant improvements in ISS life support have been proposed by the original ISS ECLSS development team.

"The baseline environmental control and life support (ECLS) systems currently deployed on board the International Space Station (ISS) and that planned to be launched in Node 3 are based upon technologies selected in the early 1990's. While they are generally meeting or exceeding requirements for supporting the ISS crew, lessons learned from years of on orbit and ground testing, together with new advances in technology state of the art, and the unique requirements for future manned missions prompt consideration of the next logical step to enhance these systems to increase performance, robustness, and reliability, and reduce on orbit and logistical resource requirements." (Carasquillo, Bagdigian, et al.)

Problems cited for the ISS ECLS include high power consumption, difficult maintainability and logistics, sensitivity of several components to particulates and fouling, gravity related problems in multi-phase fluid flow and separations, and the lack of fine particle settling in microgravity. There are potential improvements in robustness, performance efficiency, and expanded capability. These can be obtained by a more integrated design approach and by "a focused, functionally-based systems engineering approach to specifying the ECLS system and developing the process design." (Carasquillo, Bagdigian, et al.)

Beyond the need for improvements to the ISS ECLSS, Carasquillo, Bagdigian, et al. make clear the absolute necessity of on-orbit operational experience, especially over the long term. The transport and accumulation of fine particles in microgravity caused problems with ventilation fans, smoke detector, 4BMS, and the water processor 
Volatile Removal Assembly. ISS ECLSS systems were never exposed to such debris loads during development testing or flight hardware ground testing. It is necessary to acquire operational experience with ECLS equipment to identify operational failure modes and to fully understand maintainability needs.

\section{Constellation and future advanced technologies}

Technologies for life support were investigated for the Constellation program, and many could be potentially advanced as part of the overall effort to develop innovative technologies for flexible path human exploration.

\section{A. Constellation technologies}

Although Constellation life support technology development priorities for Orion and Altair were strongly focused on conforming to Constellation's constrained budget and schedule and its necessary initial focus on nearEarth missions, some of the technologies, including those being developed for Lunar Surface Systems missions should be considered for deep space life support applications. These technologies include amine-based swing bed and sorbent-based carbon dioxide removal, Bosch and electrolysis carbon dioxide reduction, thermal catalytic oxidation for trace contaminant control, advanced water disinfectants, alternative urine pretreatments, distillation and membrane-based water processing systems, an improved commode, water recovery from waste treatment, and heat melt trash compaction. (Jones and Kliss, 2010) Bosch was an SSF alternate and VPCAR a suggested non-SSF upgrade. Continuing to advance these technologies will allow NASA to build a solid technological foundation for sustainable beyond-LEO exploration.

\section{B. Advanced technologies for deep space}

NASA intends to advance the state of the art in technology areas that enable the flexible path, specifically including the critical closed loop life support system. Achieving a lower mass, more efficient, more closed, more dependable, and ultra-reliable life support system is a farsighted goal, which could support decades of continued American leadership in human space exploration. Game-changing problem-free life support is the essential technical foundation for safe, flexible, and more affordable exploration beyond-LEO to the Moon, nearby asteroids, and finally Mars.

The expertise and drive to create the necessary innovations exists in a combination of people from academic institutions, international agencies and organizations, industrial space systems groups, and NASA Centers. These diverse contributors need both support for creative research and technology development, and a sufficiently detailed common understanding of the well-integrated, dependable, increasingly self-sufficient flexible path life support system being developed.

\section{Deep space life support development planning}

A deep space life support system development effort should include the design, development, integration, testing, and demonstration of the life support system for long duration transit and planetary surface applications beyond LEO. The overall objective would be to convincingly demonstrate an operational life support capability that enables deep space exploration. The effort would include system architecture design, technology selection, component life testing, prototypes, integrated tests, and several cycles of design, long testing, and redesign. Selected components and ultimately the integrated system should be tested in the space environment under challenging conditions.

\section{A. Life support system redesign}

Both the system architecture and the subsystem technologies of the ISS should be reconsidered. The ISS life support functional architecture is a familiar general structure for the design of recycling systems, but it should be reexamined and modified as appropriate. The paired human water needs and waste streams suggest using partially separated water flows. The variability of individual human requirements and the likelihood of different crew sizes indicate that a range of processing rates will be needed, and that the final implementation should have sufficient rate adjustability to accommodate between-crew variations. Deep space missions may be transits or surface landings, so the design should be adaptable to EVA resource demands and waste handling, especially the potential high EVA cooling water use, and also accommodate possible excess propellant scavenging, the alternatives of hydrated or dehydrated food, and the potential availability of different resources on the Moon, nearby asteroids, comets, or Mars. The deep space life support system will achieve the required operational reliability using scheduled maintenance and making repairs after a failure, so it will have periods of downtime. 
Candidate enabling capabilities for flexible path closed-loop life support include:

- systems engineering models, tools and methodologies to direct integrated development efforts and optimize material and energy flows

- an air revitalization system that efficiently incorporates gas removal, separation, pressurization, storage, reduction, generation and trace contaminant control functions in a highly integrated air loop

- fully regenerative wastewater recycling systems that minimize consumables and mass, power and volume while supporting a range of mission durations

- waste processors that stabilize and render harmless human waste and trash, minimize waste volume, and manage mission byproducts to recover resources

- incorporation of biological and bioengineered processors into closed-loop systems

Advanced materials, automatic controls, and innovative design methodology should also be considered for improving system performance.

\section{B. Reducing ESM}

Significant mass savings seem to be achievable with technologies not selected for space station. For example, an overall ESM saving of up to 50 percent may be obtained using technologies that were considered for ISS. Previous estimates indicate that the two bed molecular sieve (2BMS) has lower mass than a 4BMS, a static feed water electrolysis (SFWE) oxygen generator has less mass than solid polymer water electrolysis (SPWE), and the vapor phase catalytic ammonia removal (VPCAR) urine processor has less mass than vapor compression distillation (VCD) (Jones and Kliss, 2005) The computed ESM includes only the initial mass required to provide and support the equipment, without consumables or other mass impacts. Additional ESM savings will likely be realized with other advanced technologies being developed.

\section{Increasing closure}

Water and oxygen could be either scarce or relatively plentiful on a deep space mission, depending on the variable crew size and requirements, unpredictable excess propellant scavenging, potentially large EVA water losses and EVA oxygen and cooling water demands, and the amount of water in the food. Given these variable requirements and resources, it is prudent to plan for a limited amount of water and oxygen available through resupply. Accordingly, it is necessary to develop efficient technologies for recovering oxygen, water and other resources from all possible waste and in situ sources. Recovering resources from wastes is also a useful first step in meeting potential planetary protection requirements. In addition to having the ability to achieve a high degree of closure, it is desirable to be able to vary the amount of recycling, if material sources are sufficient and demands are met. Recycling rates may have to be set higher during downtime and failure repair, or if storage buffers are being filled.

\section{Improving dependability}

The ISS was designed to be maintained over a long life by using Orbital Replaceable Units (ORUs) to replace failed components. In a detailed review of ISS WRS failures and the lessons learned, it was stated that it would be much better to be able to replace small failed components rather than large ORUs, presumably because of the difficulty of maintaining stocks of ORUs and the delay in providing them when needed. (Carter)

Analysis shows that increasing the number of components that are individually provided with spares will increase the operational reliability gained by providing a fixed mass of spares. (Jones, 2008-01-2160) Although ISS can receive replacement ORUs or components as needed, a deep space mission must carry everything along, with no further resupply from Earth. To reduce the mass of the spares, the deep space life support system must be designed for lower level part repair than ISS. However, replacing many smaller components will require more material and effort, including different types of spares, tools, test equipment, training, and crew time. (Jones, 2010) Lessons learned from the ISS life support system include understanding the capabilities of all major systems, ways to avoid specific failure mechanisms, and better approaches to maintainability and repair. These lessons from experience should be incorporated in the flexible path advanced life support system. 


\section{E. An effective integrated life support system}

As critics of NASA's newly proposed technology development emphasis have observed, advanced technologies produced in isolation will not function properly together and may have unbridgeable gaps. An integrated system design is required to produce the functionality needed by the missions. A shared understanding of the key flexible path design drivers is needed to guide and coordinate technology research and development, to ensure that the technologies can be integrated into the system without leaving holes, and to guarantee that the top level requirements are met. Providing safe, dependable, minimum cost life support requires developing an optimized, integrated, tested, demonstrated total system.

\section{F. Achieving ultra-reliability}

A major focus of deep space life support system development should be on achieving ultra-reliability. A suggested development effort has been described. (Jones and Ewert, 2010) Reliability analysis first determines the reliability requirement and allocates it to the subsystems and components. Materials, technologies, and components should be selected and designed for high reliability. Long duration testing confirms the failure rates. Systems design should segregate the failure causes in the smallest, most easily replaceable parts. The system must be designed, developed, integrated, and tested with reliability as a goal. The overall system must be tested sufficiently to identify any design errors that would preclude achieving the anticipated reliability.

There are two approaches to improving reliability, reducing the failure rate or accommodating it. The intrinsic hardware failure rate can be reduced by design and testing the components. This usually requires repeated cycles of redesign, test, operation, failure analysis, and redesign. Initial systems testing often reveals major problems that require redesign. When these are solved, many less frequently observed problems usually appear. Cutting the intrinsic hardware failure rate in half can take as much time and effort as the original system development. Achieving very high quality requires repeated redesign with significant numbers of units and long testing time.

Alternatively, a too high intrinsic failure rate can be compensated for, by providing spares and replacing failed components. Improving the intrinsic reliability is very difficult, costly, and time consuming on the scale of years. Providing spares is much more effective but increases the initial mass and launch cost for deep space missions, where all the needed spares must be sent on the mission.

Practical design exercises and reliability theory both show that ultra-reliability can usually be achieved by providing spares with a mass approximately equal to the original hardware mass. For this to be true, the original design must have a reasonable intrinsic hardware reliability, meaning at least a 90 percent chance of not failing over the mission duration. (Jones, 2008-01-2160) (Jones, 2010)

\section{Conclusion}

As NASA looks toward human missions beyond LEO into deep space, a key distinction between these missions and all crewed space missions to date is that human beings will spend longer periods of time farther from reliable logistics depots. Because of the poor economics associated with storing all supplies needed for human life support and the additional risks presented by sending logistics vehicles long distances, reliable and well-integrated closedloop life support systems will be required. These systems will need to address both the technological shortcomings and the functional integration inefficiencies of the baseline systems presently operating on board the ISS. Flexible path life support systems must incorporate regenerative technologies for process functions that traditionally have relied upon expendable resources, be more economically efficient with respect to mass, volume, and power consumption, and integrate with highly reliable, autonomous process monitoring and control systems.

Because the ISS technology selection process was focused on reducing development risk by selecting the highest TRL technologies, the technologies developed may not be the best solution for meeting the different design drivers for flexible path human exploration moving toward deep space. These drivers include the significantly higher cost of sending a kilogram of mass toward deep space, the increased needs for self-sufficiency and reliability, the ability to operate in several gravity environments, and potential requirements to support increased EVA activity, tolerate dust contamination, accommodate planetary protection measures, and take advantage of ISRU opportunities.

To meet the challenges of future flexible path human exploration, a new deep space life support system must be developed that meets these broader needs with increased system safety, reliability, maintainability, flexibility, and reparability. Developing an advanced life support system for the flexible path into deep space will be a challenging effort that may change the way we view human exploration beyond LEO, as well as find applications on Earth for treatment of water to potable standards, carbon dioxide capture and conversion, carbon sequestration, and waste minimization and co-product generation. 


\section{References}

Bagdigian. R. M., and K. Y. Ogle, "International Space Station Node 3 Environmental Control and Life Support System Status," SAE Technical Paper No. 2001-01-2387, 31st ICES (International Conference on Environmental Systems), 2001.

Carasquillo, R. L., and D. Bertotto, "ECLSS Design for the International Space Station Nodes 2 \& 3," SAE Technical Paper No. 1999-01-2146, Society of Automotive Engineers, Warrendale, PA, 29th ICES (International Conference on Environmental Systems), 1999.

Carasquillo, R. L., R. M. Bagdigian, J. F. Lewis, and J. L. Perry, "Evolution of the Baseline ISS ECLSS Technologies - The Next Logical Steps," SAE Technical Paper No. 2004-01-2385, 34th ICES (International Conference on Environmental Systems), 2004.

Carrasquillo, R. L., D. L. Carter, D. W. Holder, Jr., C. F. McGriff, and K. Y. Ogle, Space Station Freedom Environmental

Control and Life Support System Regenerative Subsystem Selection, NASA TM-4340, February, 1992.

Carter, Layne , verbal comment during "WRS Lessons Learned on ISS," teleconference presentation, January 21, 2010.

Diamant and W. R. Humphries, "Past and Present Environmental Control and Life Support Systems on Manned Spacecraft,"

SAE Technical Paper No. 901210, 20th ICES (International Conference on Environmental Systems), 1990.

Jones, H. W., "Multiple Metrics for Advanced Life Support," SAE Technical Paper No. 1999-01-2079, 29th ICES

(International Conference on Environmental Systems), 1999.

Jones, H. W., "Equivalent Mass Versus Life Cycle Cost for Life Support Technology Selection," SAE Technical Paper No. 2003-01-2635, 33rd ICES (International Conference on Environmental Systems), 2003.

Jones, H., "Life Support Dependability for Distant Space Missions," AIAA paper number 2010-01-XXXX, 40th ICES (International Conference on Environmental Systems), 2010.

Jones, H., "Lunar Base Life Support Mass Flow and Recycling," SAE Technical Paper No. 2008-01-2184, 38th ICES (International Conference on Environmental Systems), 2008.

Jones, H., "Ultra Reliable Space Life Support Systems," SAE Technical Paper No. 2008-01-2160, 38th ICES (International Conference on Environmental Systems), 2008.

Jones, H., and Ewert, M., "Ultra Reliable Closed Loop Life Support for Long Space Missions," AIAA paper number 2010-

01-XXXX, 40th ICES (International Conference on Environmental Systems), 2010.

Jones, H., and Kliss, M., "Air and Water System (AWS) Design and Technology Selection for the Vision for Space

Exploration,” SAE Technical Paper No. 2005-01-2810, 33rd ICES (International Conference on Environmental Systems), 2003.

Jones, H., and Kliss, M., "Exploration Life Support Technology Challenges for the Crew Exploration Vehicle and Future Human Missions," Advances in Space Research, 45 (2010), pp. 917-928.

Pearson, A.O., and D. C. Grana, Preliminary Results from an Operational 90-Day Manned Test of a Regenerative Life Support System, NASA SP-261, 1971.

Reed, R. D., and G. R. Coulter, "Physiology of Spaceflight," in W. K. Larson, and L. K. Pranke, eds., Human Spaceflight:

Mission Analysis and Design, McGraw-Hill, New York, undated (appeared in 2000).

Reuter, J. L., and R. Reysa, "International Space Station Environmental Control and Life Support System Status: 2000-

2001," SAE Technical Paper No. 2001-01-2386, 31st ICES (International Conference on Environmental Systems), 2001.

Wertz, J. R., and W. J. Larson, eds., Space Mission Analysis and Design, third edition, Space Technology Series, Kluwer, Dordrecht, 1999.

Wieland, P. O., Designing for Human Presence in Space: An Introduction to Environmental Control and Life Support Systems, NASA Reference Publication RP-1324, 1994.

Wydeven, T., A Survey of Some Regenerative Physico-Chemical Life Support Technology, NASA TM 101004, November, 1988. 